\title{
Linx
}

Revue des linguistes de l'université Paris X Nanterre

$11 \mid 1999$

Typologie des langues, universaux linguistiques

\section{La compréhension des syntagmes participiaux préposés sans déterminant dans les langues germaniques et spécialement en allemand}

Jacques François

\section{(2) OpenEdition \\ Journals}

Édition électronique

URL : http://journals.openedition.org/linx/892

DOI : $10.4000 /$ linx.892

ISSN : 2118-9692

Éditeur

Presses universitaires de Paris Nanterre

\section{Édition imprimée}

Date de publication : 1 décembre 1999

Pagination : 139-152

ISSN : 0246-8743

\section{Référence électronique}

Jacques François, «La compréhension des syntagmes participiaux préposés sans déterminant dans les langues germaniques et spécialement en allemand », Linx [En ligne], 11 | 1999, mis en ligne le 02 juillet 2012, consulté le 01 mai 2019. URL : http://journals.openedition.org/linx/892 ; DOI : 10.4000/ $\operatorname{linx} .892$

Ce document a été généré automatiquement le 1 mai 2019.

Département de Sciences du langage, Université Paris Ouest 


\title{
La compréhension des syntagmes participiaux préposés sans déterminant dans les langues germaniques et spécialement en allemand
}

\author{
Jacques François
}

Parmi les langues germaniques, l'allemand et le néerlandais ont maintenu, dans une large mesure (contrairement à l'anglais, mais aussi aux langues scandinaves) l'ordre sousjacent des constituants du proto-germanique, ordre "centripète mitigé » selon la terminologie de Tesnière (1959), où les membres du syntagme précèdent la tête dans les deux types majeurs de syntagmes, le nominal et le verbal. Bien qu'en diachronie le verbe fini en phrase indépendante se soit déplacé en seconde position, en subordonnée le complexe verbal demeure dans les deux langues en position finale. Si le verbe fini occupe régulièrement la position finale du complexe verbal dans les subordonnées de l'allemand - sauf en combinaison avec un verbe de modalité au passé et parfois (par analogie) au futur, cf. (1-3) - en néerlandais il passe fréquemment (cf. François 1976) en seconde (4) ou en première position (5) :

\footnotetext{
(1) all.Ich gehe einen Augenblick aus, weil das Zimmer [geputzt ${ }_{\mathrm{pp}}$ werden $_{\mathrm{inf}} \mathbf{m u} \beta$ ]. Je sors un instant parce que la pièce doit être nettoyée. (litt. nettoyée - être - doit)

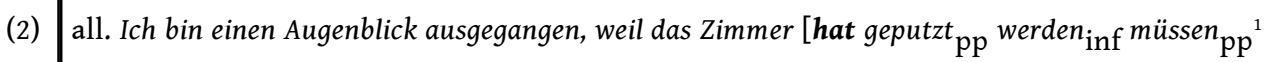
].

Je suis sorti un instant parce que la pièce a dû être nettoyée. (litt : a - nettoyée - être - dû)
} 
(3) all. Ich werde einen Augenblick ausgehen, weil das Zimmer [wird geputzt pp werden $_{\text {inf }}$ müssen inf].

Je sortirai un instant parce que la pièce devra être nettoyée.

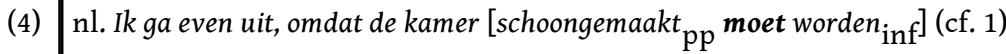

(5) nl. Ik ga even uit, omdat de kamer [moet worden $_{\text {inf }}$ schoongemaakt $_{\mathrm{pp}}$ ] (cf. 1)

2 Les constituants le plus étroitement rattachés à la tête verbale (et tout particulièrement les préverbes séparables) apparaissent en position postérieure en subordonnée (immédiatement à gauche du complexe verbal fini) comme en phrase indépendante. Dans le syntagme nominal, les syntagmes adjectivaux et participiaux à fonction déterminative ${ }^{2}$ précèdent également la tête. Lorsque les propositions relatives ont une fonction déterminative, elles sont paraphrasables par des constructions participiales insérées entre le déterminant (quand il y en a un) et le reste du SN, ex. (6)

(6) all. die [oben genannten] deutschen Fürsten

les - ci-dessus - nommés - allemands - princes

= die deutschen Fürsten, [die oben genannt wurden]

les allemands - princes - qui - ci-dessus - nommés - furent

3 Précédés d'un déterminant, ces syntagmes participiaux déterminatifs préposés sont aisément repérables. En revanche leur identification pose des problèmes intéressants pour une théorie de l'accès en compréhension à la syntaxe du syntagme nominal en allemand et en néerlandais.

\section{La présence variable de syntagmes participiaux préposés dans les langues germaniques}

\section{en allemand}

$\mathrm{Au}$ participe passé et avec déterminant, les syntagmes participiaux (abrégé désormais 'SPa') se rencontrent fréquemment, spécialement en langue administrative, ex.

(7) all. [Die [von mir bestellten] Waren] sind im Laden nicht eingetroffen.

les [par moi commandées] marchandises]

Les marchandises que j'ai commandées ne sont pas arrivées au magasin.

5 La construction est moins courante, toujours au participe passé mais sans déterminant, ex. 
(8) all. Es ist möglich,[[von mir verfaßte] Bücher] in der Bücherei zu bestellen. [Ø [par moi écrits] livres]

Il est possible de commander à la librairie des livres que j'ai écrits.

6 En allemand la construction se rencontre également au participe présent, avec ou sans déterminant :

(9) all [Ein [die Wahrheit suchender] Mensch] [un [la vérité cherchant] homme] [[die Wahrheit suchende] Menschen]] [Ø [la vérité cherchant] hommes]

\section{en néerlandais}

$7 \quad$ Les SPa au participe passé et précédés d'un déterminant sont courants, ex.

(10) nl. Het is dus ook niet te verwonderen, dat de [pas gearriverde] Dekker] (...) volkomen te goeder trouw aannam, dat [de [tegen de Jang di Pertolan uitgebrachte] beschuldigingen] op waarheit berustten ${ }^{3}$.

[le [à peine arrivé]Dekker]

[les [à l'encontre de Jang di Pertolan portées] accusations]

Il ne faut donc pas s'étonner que Dekker à peine arrivé admît en toute bonne foi que les accusations portées à l'encontre de Jang di Pertolan avaient une base véridique.

8 Comme en allemand, ils sont plus rares sans déterminant, ex.

(11) nl. de aanvallen van naburige, [onafhankelijk gebleven] stammen $\varnothing$ [voisines [indépendantes - restées] tribus]

les attaques de tribus voisines restées indépendantes

9 Mais contrairement à l'allemand les SPa au participe présent sont exclus, avec ou sans déterminant.

\section{en danois ${ }^{4}$}

Les SPa déterminatifs préposés avec déterminant ne se rencontrent qu'occasionnellement et essentiellement dans les textes administratifs, ex.

(12) dan. [De [af mig bestilte] varer] er ikke ankommet til forretningen [les [par moi commandées] marchandises] (cf. 7) 
\begin{tabular}{|l|l|}
\hline (13) dan. & $\begin{array}{l}\text { Der er mulighed for at bestille [[af mig forfattede] bøger] på biblioteket } \\
\text { [par moi écrits livres] à la librairie (cf. 8) }\end{array}$
\end{tabular}

et exceptionnels avec un participe présent précédé d'un complément référentiel, ex.

\begin{tabular}{|l|l|}
\hline (14) dan. & $\begin{array}{l}\text { [Et [sandheden og vejen søgende] menneske] } \\
\text { un la vérité et le chemin cherchant homme }\end{array}$ \\
\hline (cf. all. & Ein die Wahrheit und den Weg suchender Mensch) \\
\hline
\end{tabular}

12 Ceux-ci sont en revanche plus courants avec un complément non référentiel incorporé au participe présent, ex.

\begin{tabular}{|l|l|}
\hline (15) dan. & $\begin{array}{l}\text { et sandhedssøgende menneske } \\
\text { un vérité-cherchant homme }\end{array}$ \\
\hline (cf. all. & ein wahrheitssuchender Mensch) \\
\hline
\end{tabular}

\section{en islandais ${ }^{5}$}

13 On ne rencontre de SPa déterminatif préposé qu'avec un participe passé de préférence accompagné d'un déterminant. Si le syntagme comporte un complément d'objet, il est généralement non référentiel et souvent incorporé au participe.

\section{en anglais}

Les SPa déterminatifs préposés constituent une construction exceptionnelle. Cependant nous en avons rencontré une illustration dans un roman ${ }^{6}$ :

(16) It throws a shadow of insincerity over [their [most deeply felt] emotions]

Cela jette une ombre de duplicité sur [leurs [le plus profondément éprouvées] émotions]

\section{essai de synthèse}

Les conditions d'apparition de SPa déterminatifs préposés à une tête nominale sont résumées sur le tableau T1. Dans aucune des langues germaniques illustrées, la construction n'est courante à l'oral ni en style écrit familier. Elle concerne uniquement le style écrit administratif et académique. Les deux critères de classement des langues germaniques relativement à cette construction sont le degré d'acceptabilité du SPa au participe passé non précédé d'un déterminant et du SPa au participe présent (à complément référentiel). En fonction de ces critères, l'allemand se révèle être la langue la 
plus accueillante pour cette construction, suivie par le néerlandais, le danois, l'islandais et finalement l'anglais.

T1. Acceptabilité des SPa déterminatifs préposés dans les langues germaniques

\begin{tabular}{|c|c|c|c|c|c|c|}
\hline \multirow[t]{3}{*}{ langue } & \multirow{3}{*}{$\begin{array}{l}\text { accep- } \\
\text { tabilité } \\
\text { décrois- } \\
\text { sante }\end{array}$} & \multirow[t]{3}{*}{ oral } & \multirow{3}{*}{$\begin{array}{c}\text { écrit } \\
\text { familier }\end{array}$} & \multicolumn{3}{|c|}{ écrit administratif et académique } \\
\hline & & & & $\begin{array}{c}\text { tête: } \\
\text { PartPres }\end{array}$ & \multicolumn{2}{|c|}{ tête: PartPassé } \\
\hline & & & & & $\begin{array}{c}\text { sans } \\
\text { déterminant }\end{array}$ & $\begin{array}{c}\text { avec } \\
\text { déterminant }\end{array}$ \\
\hline allemand & $\downarrow$ & non & non & oui & oui & oui \\
\hline néerlandais & $\downarrow$ & non & non & non & oui & oui \\
\hline danois & $\downarrow$ & non & non & très rare & rare & oui \\
\hline islandais & $\downarrow$ & non & non & non & très rare & rare \\
\hline anglais & $\downarrow$ & non & non & non & très rare & très rare \\
\hline
\end{tabular}

\section{hypothèse explicative}

\section{faut à la fois}


incorporés. En anglais enfin le SAdj/SPa déterminatif est préférentiellement remplacé par une relative.

\section{La compréhension linguistique à l'écrit : composante ascendante (ou « guidée par la grammaire ") et composante descendante (ou " guidée par le savoir »)} linguistique qui sont censées modéliser aussi bien les processus de production que de réception. La réversibilité des opérations de traitement étant sujette à caution, nous ne suivrons son raisonnement que du point de vue des processus de compréhension. Dans la première hypothèse le traitement est séquentiel et strictement ascendant (du niveau acoustique au niveau conceptuel). Ce premier modèle est clairement inapte à rendre compte des observations empiriques, en particulier en termes de rapidité de réaction et d'activité dialogale. Dans la seconde hypothèse le traitement reste ascendant, mais il est conçu comme distribué : le résultat du traitement phonologique du premier segment est traité syntaxiquement tandis que le second segment est traité phonologiquement. $\mathrm{Ce}$ modèle est moins éloigné des observations, mais il ne peut pas tenir compte de l'aptitude des interlocuteurs à planifier leur réplique tout en poursuivant les processus de compréhension et fréquemment, en présence de stéréotypes, de répliquer sans attendre la fin de la réception du message. Le phénomène de la restauration phonémique, c'est-àdire l'identification spontanée dans un contexte favorable d'un mot malgré un brouillage acoustique, constitue un second argument en faveur d'un traitement non seulement distribué mais aussi « interactif », c'est-à-dire bidirectionnel (ascendant et descendant).

Dans la troisième hypothèse, que retient finalement Jackendoff, l'appariement entre les connaissances encyclopédiques et les informations extraites à la lecture du co-texte antérieur à la portion du texte en cours de lecture, produit un savoir disponible qui est immédiatement investi dans la compréhension de la suite du message par composition sélective du sens. Par ex. le lecteur d'un entrefilet sur un accident d'avion active le modèle mental ${ }^{*} \mathrm{CRASH}^{*}$ à la lecture du titre et l'apparie avec les données fournies par le début du texte : date, lieu, conditions météorologiques, nombre d'accidentés, etc. A la lecture du syntagme [la boite noire] l'interprétation «dispositif d'enregistrement des actions accomplies par le pilote » est immédiatement disponible et permet de repérer ce syntagme comme un mot composé appartenant au technolecte de l'aéronautique ${ }^{7}$.

Selon Kintsch (1995) un traitement fondé sur une attention vive au message est "guidé par la grammaire" (grammar driven), dans la mesure où les inférences y sont contraintes par la syntaxe. En revanche un traitement fondé sur le modèle mental suscité par une attention limitée aux seules unités lexicales est "guidé par le savoir" (knowledge driven), car il consiste à composer le sens du texte à partir du modèle mental et de l'interprétation (ou de l'une des interprétations) des contenus lexicaux. On peut supposer que les deux types de traitement coexistent et alternent en fonction de la cohérence de la représentation complexe qui se constitue à la lecture du texte ${ }^{8}$. Selon van Dijk \& Kintsch (1983), le mode de compréhension grammatical fonde la stratégie propositionnelle (repérage du type de procès et de ses participants), tandis que le mode de compréhension conceptuel fonde la stratégie macrostructurelle (repérage du contenu 
propositionnel par rapport à un cadre conceptuel routinier, par exemple une macroaction typique, un scénario ou script) et la stratégie superstructurelle qui consiste dans l'identification de routines textuelles'.

\section{Le degré de fiabilité de la stratégie grammaticale dans le repérage immédiat du début d'un SPa préposé en allemand}

texte suivi en allemand en auto-présentation (c'est-à-dire en cliquant à chaque fois pour faire apparaitre le mot suivant) et de décider dès que possible si cet énoncé comporte un SPa préposé. Dans ce cas il ne peut recourir à la stratégie de recherche vers l'avant des deux têtes participiale puis nominale. Reste seulement le recours combiné à la stratégie proprement grammaticale et à la stratégie « superstructurelle » (cf. \$2) d'attente de telles structures si le texte présente des caractéristiques stylistiques de caractère administratif ou académique et s'il a déjà rencontré des SPa préposés depuis le début de sa lecture (la stratégie «macrostructurelle » de Kintsch \& van Dijk 1983 paraît difficilement exploitable). Nous classons ci-après plusieurs situations typiques par ordre de fiabilité décroissante de la stratégie grammaticale pour le repérage immédiat du début d'un SPa préposé.

\subsection{Exemples de chaines permettant le recours à la seule stratégie de compréhension guidée par la grammaire}

A l'exception de rares prépositions doubles, on ne rencontre de chaîne Prep - Prep en allemand que si la première préposition introduit un SN auquel se rattache un $\mathrm{SPa}$ préposé contenant un SP complément. Dans ce cas le recours à une stratégie de 
compréhension guidée par la grammaire suffit à assurer le repérage immédiat du début du SPa à la seconde préposition. En voici un exemple :

(18) Ferrel, der als einer der fünf wichtigsten Kokainschieber Lateinamerikas gilt, war für [das Transport- und Verteilungsnetz [von [[aus Kolumbien stammendem] $]_{\mathrm{SPa}<\text { dat }}$ Kokain] $\mathrm{SN}$ ] über Guatemala und Mexiko in die USA verantwortlich. (Reuter/afp)

Ferrel qui passe pour l'un des passeurs de cocaïne les plus importants d'Amérique Latine était responsable du réseau de transport et de distribution de cocaïne provenant de Colombie vers les USA par le Guatemala et le Mexique.

litt. [le réseau de transport et de distribution [de [de Colombie provenant] cocaïne]]] - repère du début du $\mathrm{SPa}^{11}$ : aus /[von _-_

De même une stratégie de compréhension guidée par la grammaire suffit à repérer en présence d'une chaîne Prép - Adv le début du SPa à l'adverbe qui ne peut pas dépendre d'un syntagme d'un autre type, ex.

Fast eine Stunde habe er [bei $\left.\left[[\text { unerträglich dröhnender }]_{\mathrm{SPa}<\mathrm{dat}}>^{\mathrm{Musik}}\right]_{\mathrm{SN}}\right]$ an der Bar versessen... [Josef Breitbach, Das Blaue Bidet]

Il dit avoir passé près d'une heure au bar avec une musique [tonitruant intolérablement] litt. [avec [[intolérablement tonitruant] musique]]

- repère du début du SPa : unerträglich /[bei __]

La chaîne Prep - Neg permet également le repérage immédiat du marqueur de négation comme début d'un SPa (ou d'un syntagme adjectival fonctionnellement équivalent), ex. $\mathrm{SPa}<$ dat $>$ Neugier $]_{\mathrm{SN}}$ ]fragte, wunderte mich nicht. [Josef Breitbach, Das Blaue Bidet]

Que ma mère m'ait demandé des détails sur la mort de Barbe avec une curiosité [pas vraiment bienveillante], ne m'a pas étonné

litt. [avec [pas vraiment bienveillante] curiosité]]

- repère du début du SPa : nicht /[mit __]

Enfin, un substantif immédiatement suivi d'un adverbe donne immédiatement lieu à la même interprétation syntaxique, ex.

(21) Und die Dag ist auch sehr gegen solche Sachen : [das Rüberwechseln [[schlecht ausgebildeter ]$_{\mathrm{SPa} a \mathrm{gen}>} \mathrm{Kräfte}_{\mathrm{SN}}$ :gen von der einen Branche in die andere]. [H. von Fallada, Kleiner Mann, was nun ?]

Et Dag est aussi complètement opposée à des choses comme ça : le déplacement de travailleurs mal formés d'une branche vers une autre

litt. [le déplacement [mal formés<génitif>] travailleurs] d'une branche vers une autre]

- repère du début du SPa : schlecht /[Rüberwechseln _-_] 


\subsection{Exemples de repérage retardé du début d'un SPa par inférence grammaticale}

L'analyse grammaticale progressive peut fournir un indice qui permet le repérage du début d'un SPa mais avec un certain retard par comparaison avec les illustrations du §3.1. Considérons d'abord (22) qui présente un cas de coordination attendue d'un SPa préposé avec un premier participe présent préposé (repéré comme au génitif pluriel ou féminin et donc comme constituant d'un SN dépendant de la tête nominale initiale). La plausibilité du début d'un SPa est élevée à la lecture de la chaîne: préposition contractée im + substantif Schritt et devient une certitude à la lecture du pronom réfléchi sich (lequel fonctionne comme " point d'identification ultime »).

die Schlange [wartender oder [im Schritt sich zäh vorwärtsbewegender $]_{\mathrm{SPa}<\mathrm{gen}>}$ Wagen $\left.]_{\mathrm{SN}}\right]$

La queue de voitures en attente ou se déplaçant péniblement au pas

litt. [en attente ou [au pas - se - péniblement - déplaçant] voitures]

- repère du début du SPa : im (Schritt) (sich) / [wartender oder __]

(23) présente un cas différent de repérage retardé en raison du rattachement douteux de l'adverbe ungleichmäßig soit au verbe trieb (complété ultérieurement par vor sich her: repousser) soit à une structure participiale anticipée. Ici, en l'absence d'indice préalable - qui pourrait être un premier participe, par ex. ... einen Trupp erschöpfter und... (une compagnie de - épuisés et ...) - ungleichmäßig ne peut pas fonctionner comme point d'identification de la structure syntaxique et seule la lecture du participe bewaffneter <génitifs le permet.

(23) Die Schwadron (...) trieb [einen Trupp [ungleichmäßig bewaffneter] $\left.]_{\mathrm{Spa}<\mathrm{gen}>\text { Menschen }}\right]_{\mathrm{SN}}$ ] wie die Wachteln vor sich her.[H. von Hoffmansthal, Eine Reitergeschichte]

L'escadron repoussa une compagnie d'hommes inégalement armés comme des moineaux.

litt. [une compagnie - inégalement - armés<gen> hommes]

- repérage du début du SPa : le participe bewaffneter<gen>

\subsection{Un exemple d'ambiguïté persistante sur le début du SPa}

Enfin on peut rencontrer des situations où le rattachement d'un constituant demeure problématique jusqu'à la fin de la lecture de la phrase.

(24)

die liebten ja nur die begleiteten Gäste oder solche, die sich [an der Bar] [auf Kunden] [lauernde Damen] zum Mitessen und Mittrinken aussuchten oder heranbringen ließen (...) [Josef Breitbach, Das Blaue Bidet] litt. [au bar] [de clients] [étant à l'affût dames] 
(25) Trad.1 : ils n'aimaient que les clients accompagnés ou ceux qui se choisissaient au bar, pour prendre une consommation ensemble, [des dames à l'affût de clients] ou se les faisaient amener.

(26) Trad.2: ils n'aimaient que les clients accompagnés ou ceux qui choisissaient, pour prendre une consommation ensemble [des dames postées au bar à l'affût de clients] ou se les faisaient amener.

Le rattachement de [an der Bar] (au bar) soit au verbe sich...aussuchten (choisissaient) traduction (25) - soit au SPa [auf Kunden lauernde Damen] (des dames à l'affût de clients) traduction (26) - reste indécidable. En effet dans les deux hypothèses, la position du circonstant de lieu an der Bar avant un complément plus étroitement rattaché au verbe postposé est non marquée en allemand, qu'il s'agisse de auf Kunden quicomplète lauernde dans l'hypothèse de la traduction 26 ou de zum Mitessen und Mittrinken (pour manger et boire ensemble) qui complète [sich] aussuchten dans celle de la traduction 25.

\section{Conclusion}

Nous avons emprunté à l'analyse psycholinguistique de la compréhension des unités linguistiques textuelles trois terminologies qui se recouvrent plus ou moins: mode de compréhension ascendant vs. descendant (cf. Jackendoff 1987), stratégies propositionnelle, macrostructurelle et superstructurelle (cf. van Dijk \& Kintsch 1983) et compréhension guidée par la grammaire vs. guidée par le savoir (cf. Kintsch 1995) .

Sur la question du point d'identification du début d'un SPa préposé sans déterminant en allemand, la distinction la plus pertinente est la dernière, combinée avec la stratégie superstructurelle (prise en compte du type de texte à analyser). En effet, compte tenu de la nécessité de garder en mémoire de travail l'anticipation de deux têtes syntaxiques, un participe tête du SPa et un substantif tête du SN régissant ce SPa, le traitement ne peut être rapide (sans reconstruction, saut en avant ni retour en arrière) que s'il est à la fois guidé par la grammaire (analyse grammaticale gauche-droite et haut-bas selon Kimball 1973, c'est-à-dire par anticipation d'une hiérarchie de nœuds dominants jusqu'à consolidation de la structure par confirmation de ces nœuds) et par une attention vive aux indices de début possible d'un SPa (stratégie de recherche guidée par le savoir acquis sur le type de texte analysé).

Dans la situation expérimentale que nous avons imaginée au \$3, le lecteur est astreint à prendre rapidement une décision d'identification d'un début de SPa préposé à partir d'un traitement strictement progressif. Seules les procédures évoquées ci-dessus sont alors à sa disposition. Dans une situation plus conforme à la réalité de la lecture, on peut supposer qu'il procédera fréquemment à des traitements de vérification et en particulier à des saccades oculaires vers l'avant afin de repérer les deux têtes participiale et nominale anticipées (selon le mode d'analyse syntaxique haut-bas de Kimball 1973), et vers l'arrière pour vérifier la bonne formation de la structure syntaxique ainsi obtenue.

Le recours à de tels traitements anticipateur et vérificateur - sans doute éminemment variable selon différents paramètres, entre autres : les sujets, les structures à analyser et 
l'importance sémantique du contenu du SPa pour la compréhension de la phrase qui le contient et de la place de celle-ci dans le texte - devrait pouvoir être testé à l'aide des méthodes d'analyse des mouvements des yeux durant la lecture (cf. Rayner \& Sereno 1994). Seule une telle méthode nous parait apte à éclairer la question de l'aptitude des sujets germanophones (natifs ou non) à repérer le début d'un SPa préposé et à traiter sa structure interne et son rattachement dans les limites de leur mémoire de travail, avec pour hypothèse complémentaire que l'exploitation de celle-ci est fonction du degré d'attention, lequel est lui-même dépendant de l'appréciation par le lecteur des chances de rencontrer des structures de ce type dans le texte qu'il est en train de lire, c'est-à-dire dont il cherche à extraire des informations.

\section{BIBLIOGRAPHIE}

\section{Références}

BERNICOT, J. (1994) L'acquisition du langage : étapes et théories. In : R. Ghiglione \& J.F. Richard (eds.). Cours de psychologie Tome 3 : Champs et théories. Dunod/CNED

CREISSELS, D. (1995) Eléments de syntaxe générale. Paris : P.U.F.

FRANÇOIS, J. (1976) Zu einer generativen Intersyntagmatik. Die Stellung der Hilfsverben im deutschen, französischen und niederländischen Nebensatz. In : K. Braunmüller \& W. Kürschner (Hrsgg.) Grammatik, Akten des 10. Linguistischen Kolloquiums, Tübingen 1975, Band 2. Tübingen : Niemeyer, 187-198.

Gosselin, L. (1997) Les études psycholinguistiques sur la compréhension des expressions ambiguës : une critique linguistique. In : J. François \& G. Denhière (eds.), Sémantique linguistique et psychologie cognitive. Grenoble : P.U.G., 75-115.

JACKENDOFF R. (1987) Consciousness and the computational mind. Cambridge (Mass.), M.I.T.Press [spécialement le chapitre $6:$ Language processing]

KIMBALL, J. (1973) Seven principles of surface structure parsing in natural language. Cognition 2 : 15-47.

KINTSCH, W. (1995) How readers construct situation models for stories : The role of syntactic cues and causal inference. In M.A. Gernsbacher \& T. Givon (eds.) Coherence in spontaneous text. Amsterdam : Benjamins, 139-160.

LeHMANN, C. (1995) Relativsätze. In A. von Stechow \& W. Sternefeld (Eds.) Syntax, ein Handbuch. Berlin : De Gruyter, 1199-1215.

LEMARECHAL, A. (1997) Zéro(s). Paris : P.U.F.

Petursson, M. / Eyjolfsson, E. (1996) Manuel d'islandais. Paris : Klincksieck.

RAYNER, K. / SERENO, S. (1994) Eye movements in reading, Psycholinguistic studies. In : M.A. Gernsbacher (ed.) Handbook of psycholinguistics (Chapter 3). New-York : Academic Press, 57-81. 
ROULin, J.L. \& MONNIER C. (1996) La mémoire de travail. In : F. Eustache, B. Lechevalier, F. Viader (eds.), La mémoire, Neuropsychololgie clinique et modèles cognitifs. Bruxelles : De Boeck, 237-278.

SWINNEY, D. (1979) Lexical access during sentence comprehsneion : (Re)consideration of context effects. Journal of Verbal Learning and Verbal Behaviour, 18 : 645-659.

TESNIERE, L. (1959) Eléments de syntaxe structurale. Paris : Klincksieck.

VAN DIJK T. \& KinTSCH W. (1983) Strategies in discourse comprehension. New-York : Academic Press.

\section{NOTES}

1. Dans cette construction les verbes de modalité ont une forme de participe passé identique à leur forme d'infinitif.

2. Ces syntagmes réalisent un mode de l'opération de 'relativisation', cf. Creissels 1996 : 316-323, Lehmann 1995, Lemaréchal 1997, Chapitre 3 : Zéro et les relatives.

3. Mulatuli, Max Havelaar, Inleiding, p. vii, Antwerpen, De Nederlandsche Boekhandel, 1965

4. Les données du danois, aimablement fournies par le Professeur Lisbeth Falster-Jakobsen, valent mutatis mutandis pour le suédois et le norvégien.

5. Nous remercions Mme Lebreton, lectrice d'islandais à l'Université de Caen, pour ces données. Le Manuel d'islandais de Petursson \& Eyjolssson (1996) est pratiquement muet sur ce point.

6. Somerset Maugham, The moon and sixpence, p.55

7. Cependant Jackendoff, convaincu par l'expérience de Swinney (1979) sur les conditions de résolution des ambiguités lexicales en contexte, demeure fidèle à une conception modulariste qui suppose une activation simultanée des sens associés dans le lexique mental au lexème à traiter, laquelle serait suivie à moins de $400 \mathrm{~ms}$. de l'élimination du sens le moins compatible avec le traitement du co-texte (composition sélective du sens de la proposition). On peut se reporter à Gosselin (1997) pour une discussion de cette expérience.

8. On sait par ex. que le sens des phrases passives et des relatives dont le pronom occupe la fonction d'objet et dont le sujet est postposé au verbe est bien identifié par les enfants dès 3 ans 7 mois si les référents du sujet et de l'objet appartiennent à une classe conceptuelle clairement distinctible qui leur confère respectivement le statut d'agent et de patient prototypique de l'action exprimée (mode de compréhension guidé par le savoir). Dans le cas inverse, la compréhension (nécessairement guidée par la grammaire) ne devient assurée que bien plus tard, à l'âge de 10 ans 6 mois (cf. Bernicot 1994 :416-7).

9. Il est à noter que ces routines textuelles peuvent être identifiées au cours de la lecture, ce qui est important pour notre propos. En effet la récurrence de syntagmes participiaux déterminatifs préposés entraîne sans doute une attention plus vive aux structures syntaxiques dans lesquelles ils apparaissent plus ou moins régulièrement.

10. La compréhension d'un syntagme participial préposé peut conduire à un traitement syntaxique du type désigné dans la littérature psycholinguistique comme " garden-path ", c'està-dire produisant un arbre syntaxique incohérent.

11. En reprenant une terminologie appliquée en psycholinguistique (W. Marslen-Wilson) à l'identification des unités lexicales à l'oral, on pourrait qualifier le repère du début du SPa préposé de « point d'identification » c'est-à-dire de morphème à la lecture duquel l'identification de la connexion syntaxique dominante peut se faire par anticipation. 


\section{AUTEUR}

\section{JACQUES FRANÇOIS}

Université de Caen, Département de Linguistique \& CNRS, UPRES-A 6047 ELSAP 